\title{
A Scanning Electron Microscopic and Quantitative Histologic Description of Lens Regeneration in the Newt, Notophthalmus viridescens
}

\author{
THOMAS G. CONNELLY \\ Department of Anatomy, and Center for Human Growth and Development, \\ The University of Michigan, Ann Arbor, Michigan 48109
}

\begin{abstract}
Adult newts (Notophthalmus viridescens) were lentectomized and at intervals from 4 to 21 days after lentectomy iridocorneal complexes from these animals were examined by scanning electron microscopy to allow a full appreciation for the shape of the regenerating lens. Until around day 12 after lentectomy the posterior surface of the iris is covered by a dense mat of fibrous material which cannot be removed without damage to the iris and which obscures the events of cytoplasmic shedding. The regenerate becomes visible first around stage IV (day 12). A small but clear groove demarcates the regenerate from the rest of the iris. As regeneration progresses there is a marked reduction in debris on the iris surface and the regenerate appears as a U-shaped thickening occupying about one-third of the dorsal half of the iris. During later stages (VI-X) the regenerate protrudes into the pupil inferiorly and posteriorly towards the retina, but does not encroach laterally on the remaining pigmented iris tissue. Prior to secretion of the lens capsule the outline of individual cells is visible on the surface of the regenerate and some regenerates exhibit a prominent dimple on their posterior aspects. Following secretion of the capsule the surface of the regenerate becomes smooth. Quantitative studies show that volume and maximum section area of the regenerate are both more strongly correlated with developmental stage of regeneration than with time after lentectomy.
\end{abstract}

Lens regeneration is a process which involves the metaplastic transformation of pigmented epithelial cells at the dorsal pupillary margin of the iris into lens fiber cells (Reyer, '71). This process has been studied extensively at the biochemical and ultrastructural levels (Yamada, '67, '72, '77, for complete review). A complete series of morphological stages in lens regeneration has been described histologically (Sato, '40; Reyer, '48; Yamada, '67), and in most studies involving morphological determination of lens regeneration it is the histological description which is presented. Stone and Steinitz ('53) described the gross morphology of the lens regenerate and correlated the macrostructure with the histological events occurring during the production of a new lens. The gross dissections provide a clearer picture of the full extent of the area of dorsal iris involved in lens regener- ation than do the representative sections upon which the staging systems are based. Scanning electron microscopy does not allow one to visualize the transparency of the developing lens, but it does provide superior detail of the surface changes which occur in lens regeneration as well as an excellent appreciation of the gross structural changes which occur during the maturation of the new lens vesicle.

Although several authors have described the growth of the lens regenerate these studies have utilized only the dimensions of representative median sections as indicators of growth. In the present study the growth of the lens regenerate as a function of time and of developmental stage was followed by calculating the volume of several lens regenerates of various ages. Thus, the results presented below correlate surface morphology, histology and growth of the regenerating lens. 


\section{METHODS}

Adult newts (Notophthalmus viridescens) obtained from Bill Lee's Newt Farms (Oak Ridge, Tennessee) were anesthetized in ethyl$\mathrm{m}$-aminobenzoate methane sulfonate (EAMS, Eastman; 1:1,000) and subjected to bilateral lentectomy. At regular intervals from 4 to 21 days after lentectomy iridocorneal complexes (ICC's; Connelly et al., '73) from these animals were fixed in $3 \%$ glutaraldehyde in $0.1 \mathrm{M}$ phosphate buffer (Dumont and Yamada, '72). Several ICC's from unoperated animals were also fixed for determination of normal iris and lens morphology. Fixed specimens were dehydrated through graded ethanols to $100 \%$ ethanol and then carried through the $\mathrm{CO}_{2}$ critical point drying method in a modified Denton Vacuum Critical Point dryer. Following drying ICC's were mounted on aluminum stubs with Duco cement, coated with gold and examined with a JEOLCO JSM-U3 scanning electron microscope at the University of Michigan Scanning Electron Microscope and Electron Microprobe Laboratory.

Some specimens which had been scanned were removed from stubs, rinsed in absolute acetone, infiltrated under vacuum in Spurr low viscosity resin (Polysciences) and sectioned serially in the nasotemporal direction at $1 \mu \mathrm{m}$ thickness on a JB-4 rotary microtome using glass knives. Only every tenth section through the regenerate was saved. Sections were floated on drops of water on subbed slides so that the serial organization of the sections could be maintained. After sections had dried they were stained with $1 \%$ methylene blue in $1 \%$ borax, examined and photographed with a Zeiss compound light microscope.

For quantitative measurements 30 newts were bilaterally lentectomized and placed in an incubator at $25^{\circ} \mathrm{C}\left( \pm 0.2^{\circ} \mathrm{C}\right)$. A 12 -hour light/dark cycle was maintained in the incubator. At 2-day intervals from day 12 to day 22 post-lentectomy five animals from this group were decapitated and their heads and eyes fixed in Bouin's fluid. Eyes were dissected free from the heads, embedded in Paraplast and serially sectioned at $10 \mu \mathrm{m}$ thickness in the nasotemporal direction. The stage of regeneration attained by each of the 55 eyes ultimately available for study were recorded. Twenty-five of those 55 lens regenerates were also measured to calculate regenerate volume. The area of the regenerate in each section was determined with the aid of a Quantimet 720 Image Analyzer. The volume of the regenerate was calculated by summing
Abbreviations
A. Anterior chamber
N, Nasal axis
D, Dorsal
F. Lens fiber core
L, Lens
P, Pupillary margin
$R$, Regenerate
$\mathrm{S}$, Iris stroma
M, Presumed macrophage
T, Temporal axis

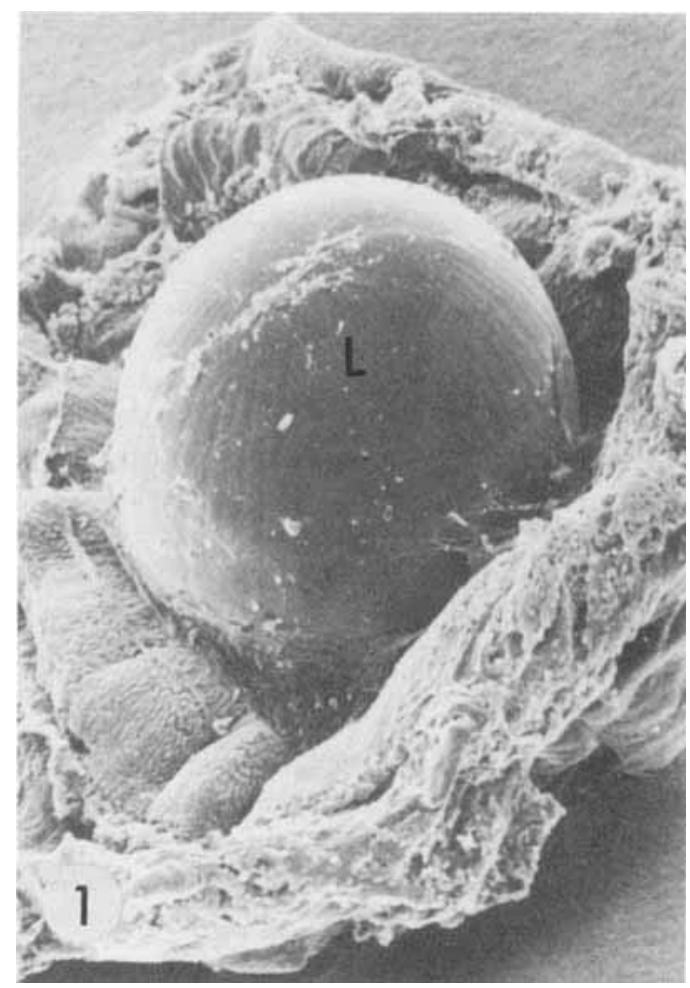

Fig. 1 A view of the retinal aspect of an iridocorneal complex with the lens still in position. The striations in the lens surface are visible and one can also see the fibers of the suspensory ligament where lens and iris come in close proximity. $\times 72$.

Fig. 2 A view of the retinal surface of a normal iris. The cells are elongated and almost rectangular in shape. Note the cobblestoned appearance of the surface of these cells. $\times 3,600$.

Fig. 3 A scanning electron micrograph of an early stage IV regenerate. Many elongated cells with prominent processes can be seen on the surface of the regenerate, and some of these cells appear to send processes deep into the regenerate. The groove separating the regenerate from the rest of the iris is indicated by the asterisk. In some places flattened cells send processes across the groove. The histological appearance of this regenerate is shown in figure $4 . \times 600$.

Fig. 4 A light micrograph through the regenerate in figure 3 . The asterisk indicates the groove marked in figure 3 . It is clear from these figures that the histological preparations give very little feeling for the amount of cellular activity which is going on on the regenerate surface, and which is a prominent feature of the scanning electron micrographs. $\times 480$. 

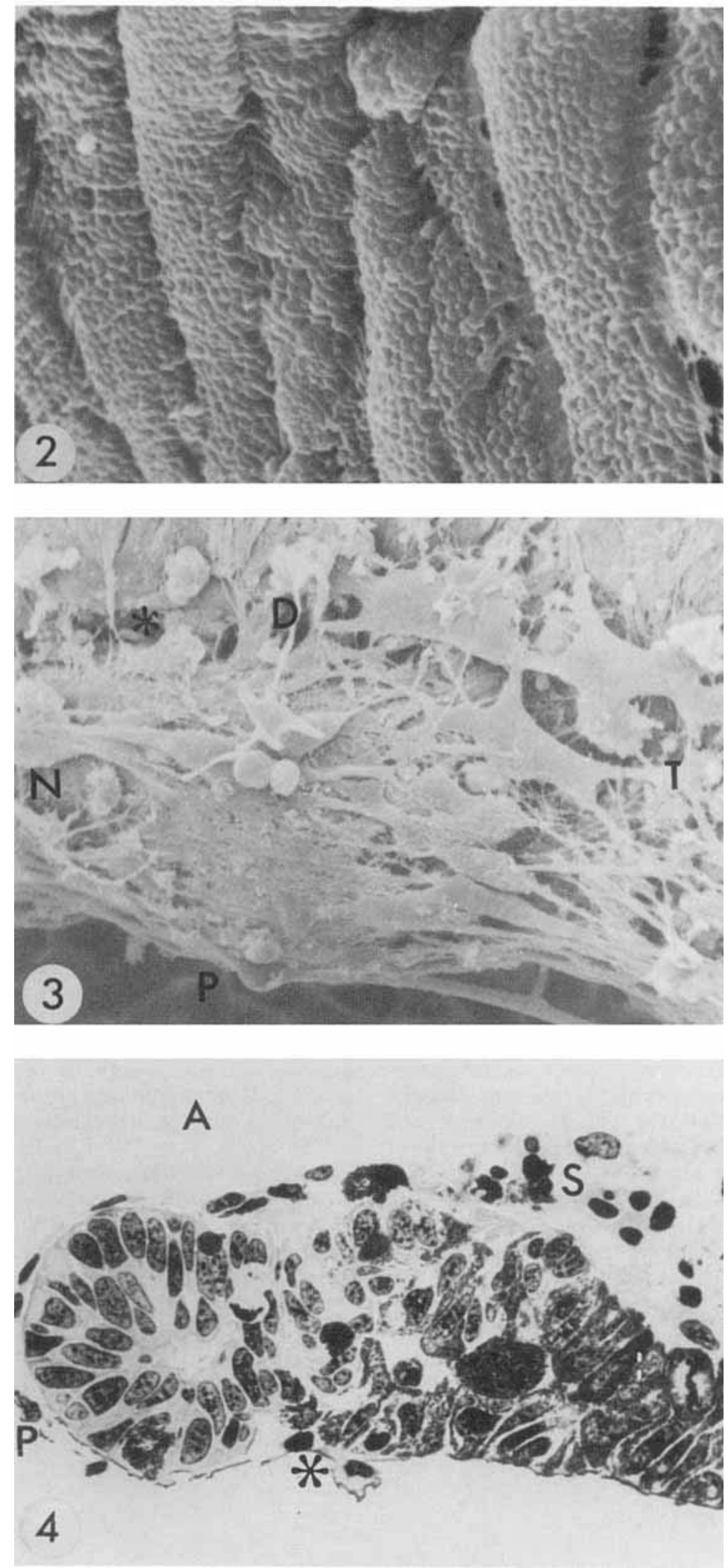


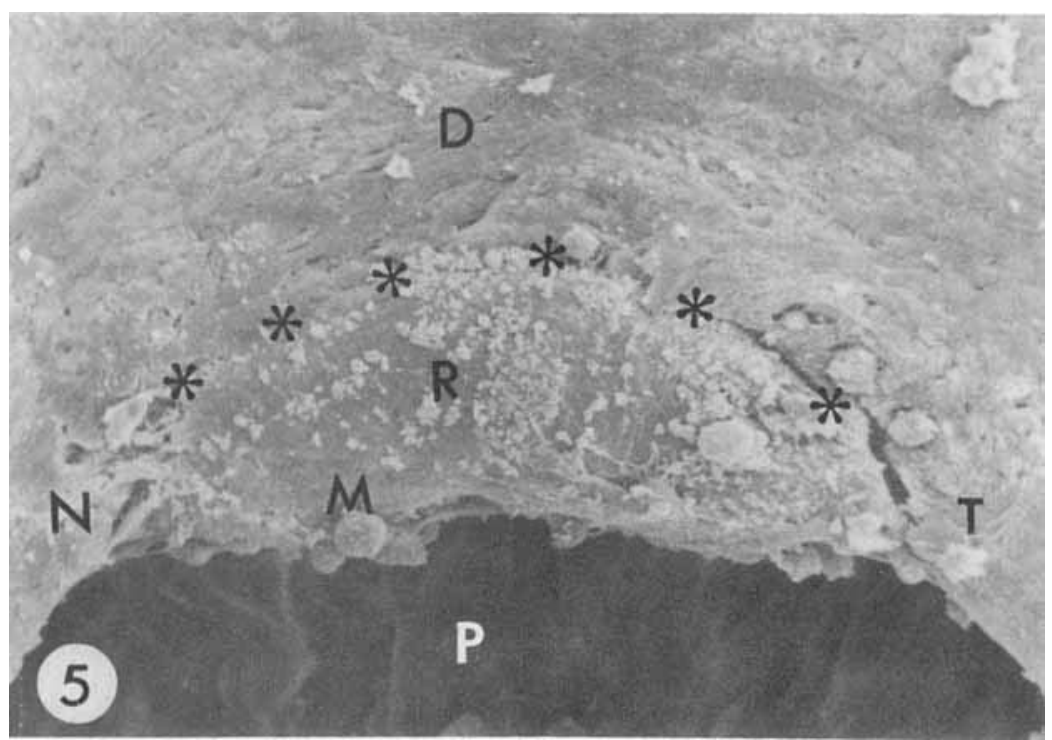

Fig. 5 A scanning electron micrograph of a regenerate (stage $V$ ) which clearly illustrates the extent of the groove (asterisks) which delimits the regenerate. $\times 475$.

the total areas of all sections of the regenerate and multiplying by the section thickness. The results obtained were described statistically using the MIDAS statistical package of the University of Michigan Statistics Research Laboratory.

\section{RESULTS}

Figure 1 shows the retinal surface of a normal iridocorneal complex with the lens still attached. The surface of the lens is smooth but longitudinal striations of marked regularity are also visible. The iris epithelial cells are arranged circumferentially around the pupil and the surface of the cells appears cobblestoned (fig. 2).

Following lentectomy the events of regeneration occur at a somewhat varied rate within a sample population (fig. 12). For example, one can expect to find at least a 2.5-stage range of regeneration at almost any given time after the process has begun (Yamada and Roesel, '64; Connelly et al., '73; Reyer, '54; Stone and Steinitz, '53). Therefore, the description which follows concentrates more on the developmental rather than the chronological time scale.

By four days after lens removal a dense feltwork of cells and fibers covers the inner aspect of the iris epithelium. This mat obscures most of the surface morphology of the iris and is impossible to remove without damage to the iris until much later in the process. Most of the round cells visible on the iris surface are probably macrophages which enter the area in response to ocular trauma. Although many events occur in the iris during the first 10 to 12 days after lentectomy, including cytoplasmic shedding, the persistence of the fibrous feltwork adherent to the inner iris lamina prevents the visualization of these events with the scanning electron microscope. By 12 days after lentectomy the fibrous mat becomes less closely applied to the epithelium and can be removed by careful dissection. The middorsal region of the iris now shows distinct signs of a developing lens (fig. 3). Histological examination of this regenerate shows that it has already attained stage IV of Yamada ('67), (see fig. 4). Stage IV regenerates can be found as late as 16 days after lentectomy.

The stage $\mathrm{V}$ to $\mathrm{VI}$ regenerates appear as clearly visible structures distinct from the rest of the iris. There appears to be a groove at the dorsal border of the regenerate which separates it from the rest of the iris (fig. 5) and which is also visible histologically. The histological picture, however, gives no appreciation of the full extent of this groove laterally nor of its prominence in outlining the regenerate. As Stone and Steinitz ('53) noted, stage VI is not a clearly defined stage and should be distinguished externally from stage $\mathrm{V}$ only by an increase in size. 

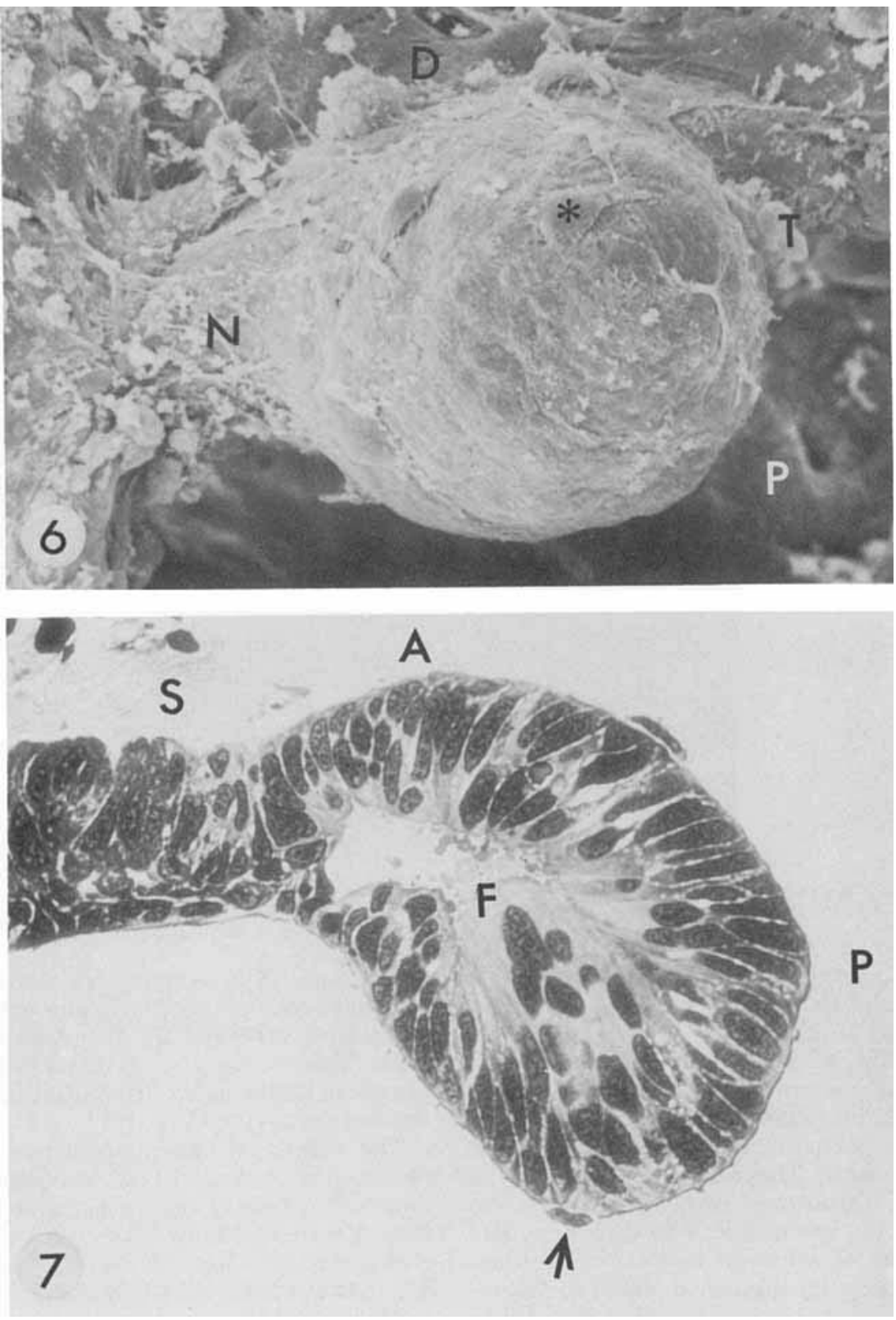

Fig. 6 A scanning electron micrograph of a stage VII regenerate. This regenerate is beginning to protrude deeper in to the pupil and towards the vitreous body. Note the clear demarcation of the regenerate. Numerous cells are visible on the surface of the dorsal iris tissue as well as on the regenerate itself. The cell marked with an asterisk is visible in figure $7 . \times 475$.

Fig. 7 A light micrograph of a section through the regenerate in figure 6 . Note the formation of the lens fiber hillock which is bulging into the lumen of the lens vesicle. The cell marked by an arrow is the same one indicated in figure $6 . \times 600$. 


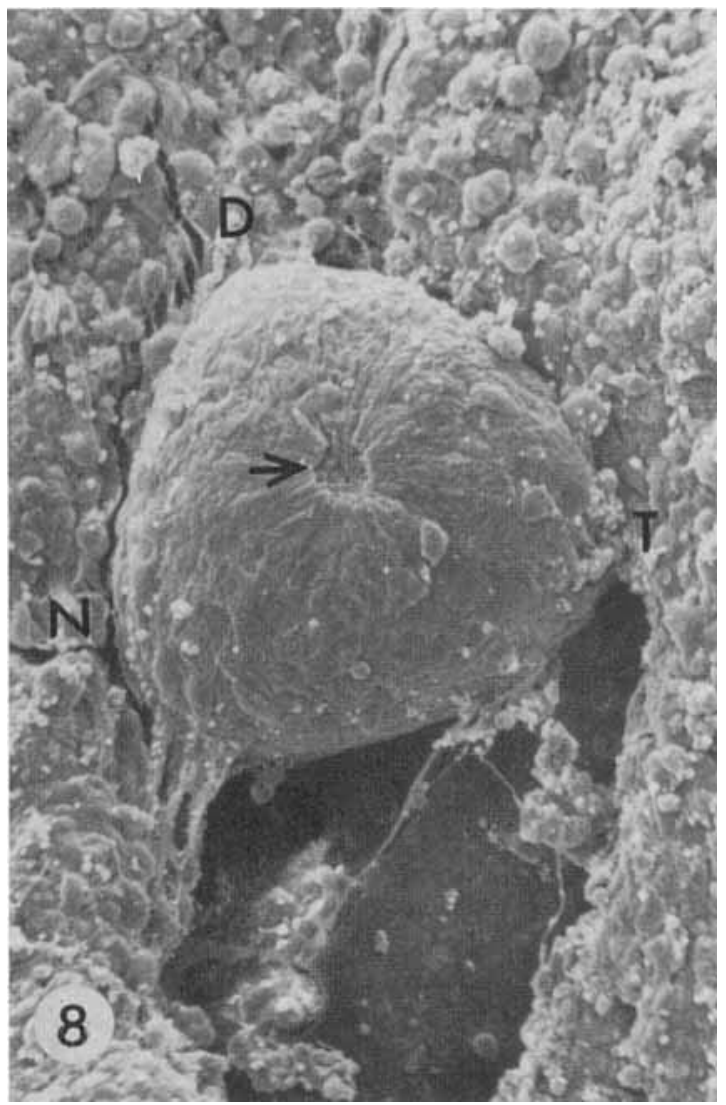

Fig. 8 A scanning electron micrograph of a stage VI-VII regenerate which displays a prominent dimple on its retinal surface (arrow) $\times 300$.

Stage VII regenerates are distinguished histologically by the formation of the lens fiber hillock (fig. 7). Externally the stage VII regenerate (fig. 6) appears to be more prominently bulbous, protruding further from the dorsal iris. This protrusion is directed towards the retina, particularly in the middle of the mid-dorsal zone. There is a decrease in the amount of fibrous and cellular debris on the surface of the iris and lens at this stage and the outlines of some cells of the regenerate can be clearly distinguished. Some of the regenerates in the range of stages V-VII show a prominent dimple on the posterior aspect of the lens (fig. 8).

In stage VIII-IX regenerates the fiber core takes up increasingly more space in the lens vesicle (fig. 9). The surface of the lens becomes smoother (fig. 10) and the outlines of individual cells become less visible. The connection of the lens with the rest of the dorsal iris becomes constricted medio-laterally.
In stage IX-X regenerates the secondary lens fiber cells progressively surround the primary fiber core and the lens capsule can be seen. This is manifested externally by the complete obliteration of cell outlines on the regenerate surface (fig. 11).

The results of the quantitative measurements show that there is a high correlation between the stage of regeneration attained and time after lentectomy $(R=0.92)$ in the general population (fig. 12). It is also clear (fig. 12) that at almost all times examined there is a range of 2.5 to 4.5 stages within each group. The correlation between stage of regeneration and time after lentectomy was somewhat lower in the population used for quantitative measurements (fig. 13; $R=0.88$ ) it is still sig. nificantly high. The relationship between regenerate volume or maximum section area and time after lentectomy is depicted in figure 14. Figure 15 demonstrates the relationship between volume of the regenerate or max- 

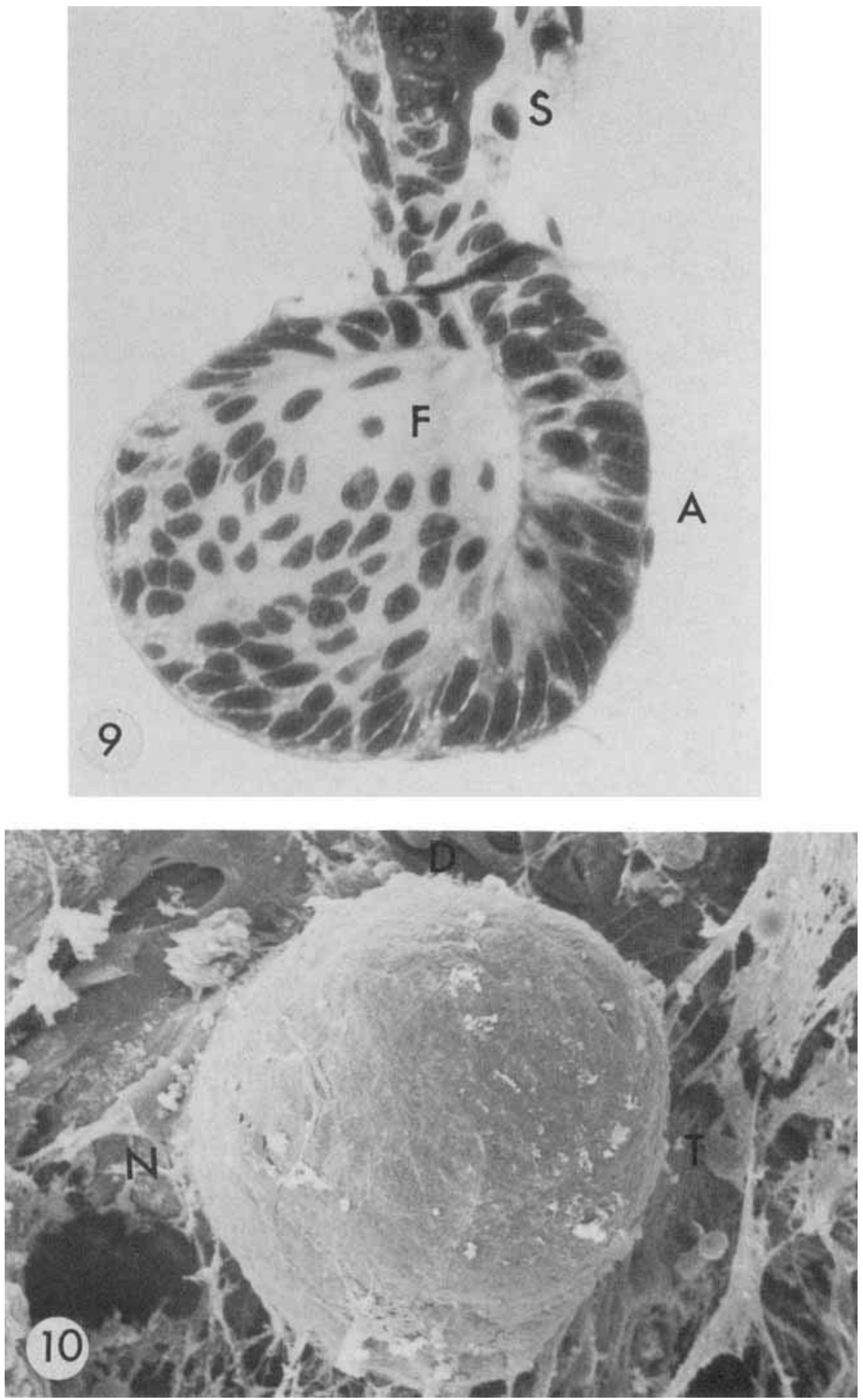

Fig. 9 A light micrograph of a stage VIII-IX regenerate which is also depicted at the SEM level in figure 10. Note the prominent lens fiber core which in this section is obliterating the lumen of the lens vesicle. In other sections through the regenerate one can still see the open lumen. $\times 475$.

Fig. 10 A scanning electron micrograph of the regenerate shown histologically in figure 9. The only marked difference between this regenerate and those of earlier stages is an increase in size and a smooth appearance of its surface. This probably reflects the thickness of the lens capsule which is beginning to form. It is clear that the histological description of the regenerates indicates much more about their differentiation than does the surface view. $\times 600$. 


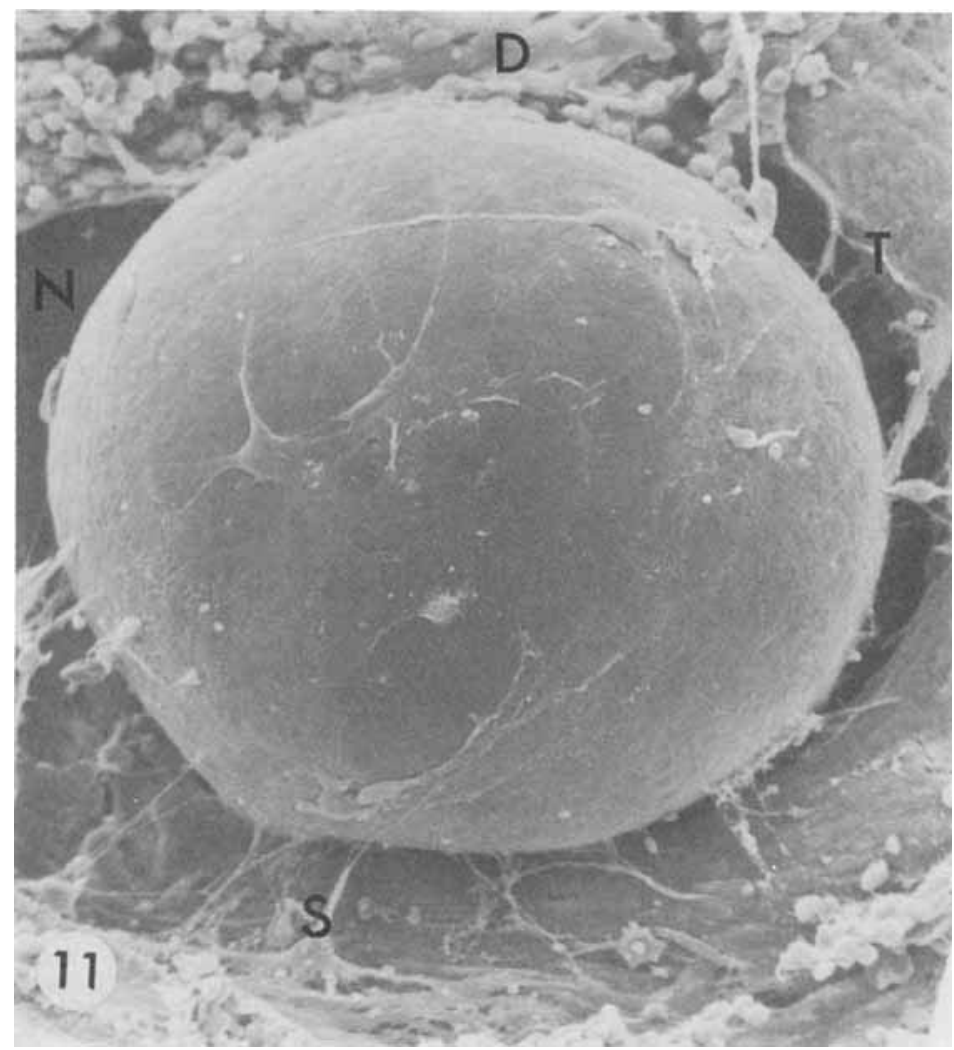

Fig. 11 A scanning electron micrograph of a late stage X or XI regenerate. Note the smooth appearance of the lens surface indicating that the lens capsule has fully formed. Some squamous cells can still be seen on the surface of the regenerate. Fibrous connections between the new lens and the iris can be seen in the lower portion of the micrograph. The meridional striations of the mature lens have not yet become visible. $\times 360$.

imum section area and stage of regeneration. These two figures show that in both cases there is a higher correlation between either of the measured variables and developmental stage rather than time after lentectomy. Figure 16 shows the correlation between maximum section area and regenerate volume.

\section{DISCUSSION}

It was hoped that a study of lens regeneration with the scanning electron microscope would enable us to visualize the events of cytoplasmic shedding associated with dedifferentiation of iris epithelial cells. However, as the results presented here show, this process is obscured by the dense feltwork of fibers and debris present on the retinal surface of the iris following lentectomy. It is probable that this feltwork represents remnants of the suspensory ligament (Higginbotham, '77; and personal communication). The results of our SEM study reveal in some detail the extent of the regenerative process laterally and dorsally within the iris. A prominent feature evident in our micrographs which is not clear in earlier studies is the amount of cellular activity (presumably from macrophage influx) occurring on the surface of the regenerates even during later stages when cytoplasmic shedding has ceased.

One of the more interesting features revealed by the SEM which has not been described previously is the dimple in the regenerate surface which appears on the retinal aspects of the regenerate during stages V-VII. It is interpreted as being the point at which lens epithelial cells involute toward the fiber core in the process of lens growth and their transformation into lens fiber cells. This dimple later becomes obscured by the lens capsule.

The results of quantitative studies of the regenerating lens provided some interesting information. Experimental analysis of problems in regeneration has been hampered by 

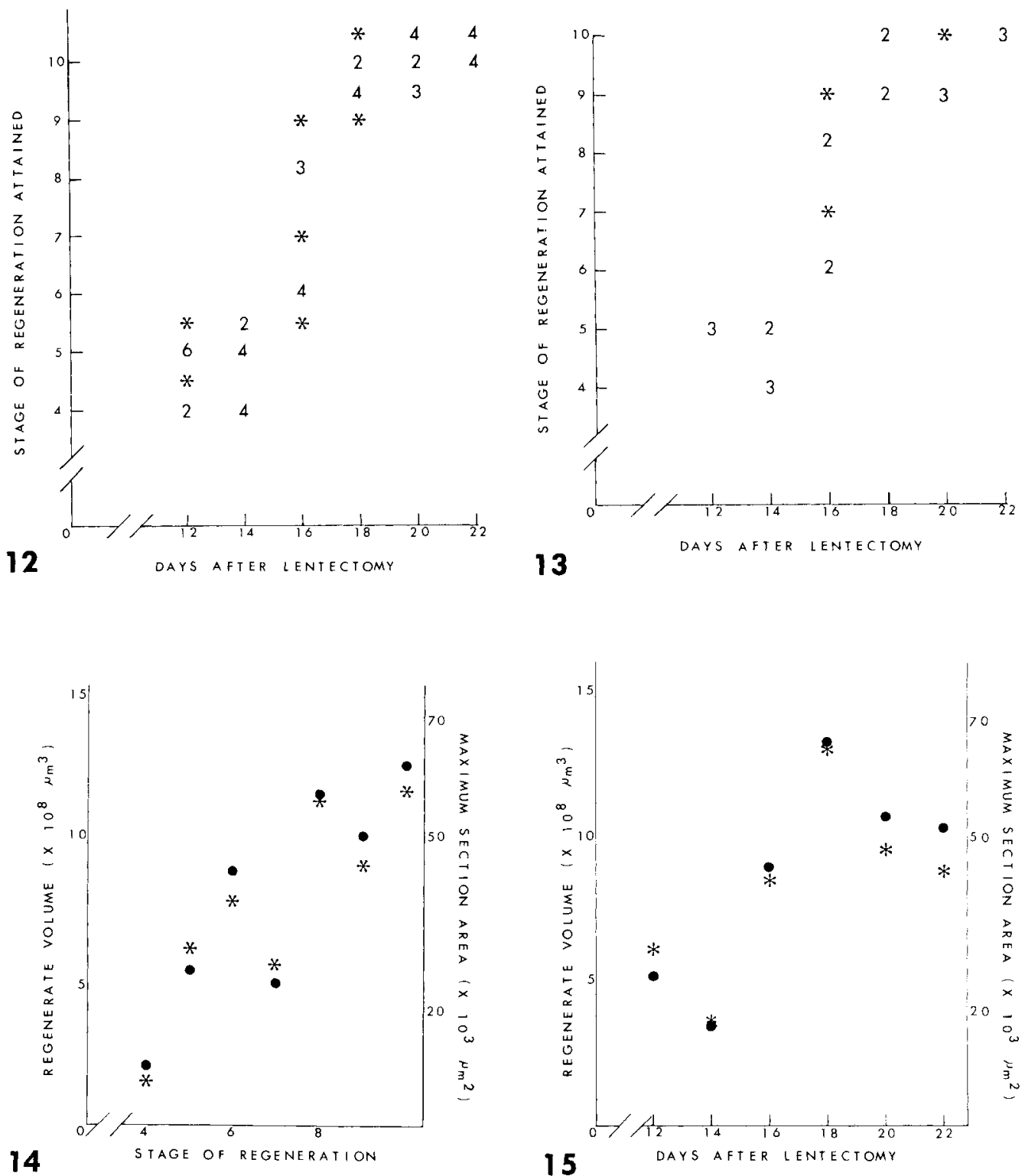

Fig. 12 A scatter plot of the stages of regeneration attained as a function of time after lentectomy in a population of 30 newts. Fifty-five regenerates were studied. The numbers indicate the number of regenerates at a given stage at a particular sample point. The asterisks indicate single regenerates. Note that there is much overlap between time points. The correlation coefficient between stage of regeneration and time after lentectomy in this population is high $(R=$ 0.92).

Fig. 13 A scatter plot of the stages of regeneration attained as a function of time after lentectomy in the population used for quantitative purposes. Twenty five regenerates were studied. The numbers indicate the number of regenerates at a given stage at a particular sample point. The asterisks indicate single cases. Although there is less overlap between time points in this group the correlation coefficient between stage of regeneration and time after lentectomy is lower ( $R$ $=0.88$ ) than that for the general population. This correlation coefficient is still significantly high to indicate an almost linear relation between the two variables.

Fig. 14 A plot of mean regenerate volume (*) or mean maximum section area ( ) as a function of time after lentectomy. The correlation coefficient between volume and time is 0.42 and between area and time is 0.60 .

Fig. 15 A plot of mean regenerate volume $\left(^{*}\right)$ and mean maximum section area $(\bullet)$ as a function of developmental stage. The correlation between volume and stage is 0.64 and between area and stage is 0.78 . 


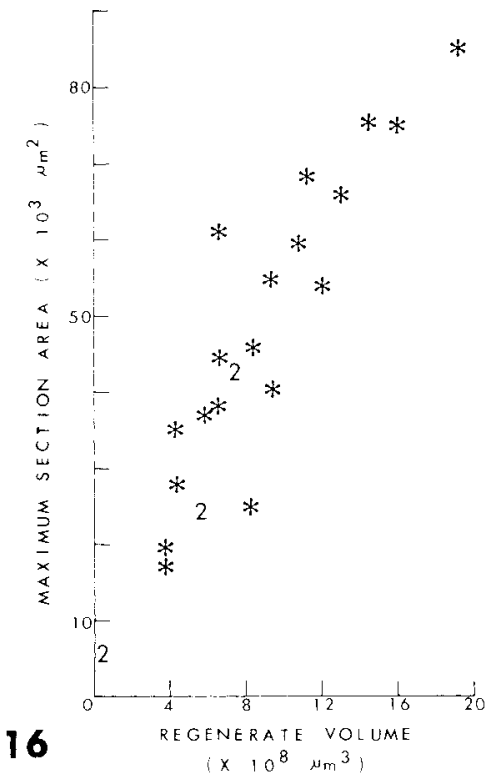

Fig. 16 A plot of maximum section area versus regenerate volume. The correlation coefficient between these two variables is 0.95 .

the variability in regeneration progress in vivo. The 2 - to 3 -stage variation usually visible at times between 12 and 22 days makes statistical evaluation of results difficult. It appears that similar variability occurs in growth of the regenerate when measured as an increase in volume or as an increase in the size of the largest histological section through the regenerate. Thus, a shift to measuring the volume or maximum section area would appear to be of little value in most in vivo studies unless one were convinced that experimental intervention was causing a block in redifferentiation but allowing the continuation of production of depigmented cells. If one were to use some measurable parameter as an indicator of lens progress maximum section area would appear to be the one of choice since it correlates better with both stage and time post-lentectomy than does volume. It is probable that the reason for this closer correlation is the fact that the stages of lens regeneration are based on the appearance of the median section through the regenerate. This is usually the one of greatest area. It is also probable that the modest degree of correlation between maximum area and regeneration stage is an indication that factors other than size must also be considered when assigning a regenerate to a given morphologic stage. The presence or absence of fiber cell elongation, the size of the fiber hillock, the closure of the lumen of the lens vesicle in later stages are all examples of such factors. The present results extend earlier quantitative studies by Reyer ('48), Zalokar ('44) and Stone and Steinitz (53), and confirm the value of their use of measurements on median sections through the regenerates as an estimate of regenerate growth. The measurements presented in this study differ from theirs in that we have also included the depigmented areas within the iris as well as those within the lens vesicle.

\section{ACKNOWLEDGMENTS}

The author would like to thank Ms. Elizabeth K. Ashcraft and Ms. Rosalie A. Beer for their excellent technical assistance. This work was supported partially by a grant (HD-08150) from the USPHS-NIH. Thanks are also due to Doctors B. M. Carlson and Tuneo Yamada for critically reviewing the manuscript.

\section{LITERATURE CITED}

Connelly, T. G., J. R. Ortiz and T. Yamada 1973 Influence of the pituitary on Wolffian lens regeneration. Develop. Biol., 31: 301.315.

Dumont, J. N., and T. Yamada 1972 Dedifferentiation of iris epithelial cells. Develop. Biol., 29: 385-401.

Higginbotham, L. L. 1977 The redevelopment of the zonular apparatus during lens regeneration in the newt eye. Anat. Rec.,187: 604.

Reyer, R.W. 1948 An experimental study of lens regen. eration in Triturus viridescens viridescens I. Regeneration of a lens after lens extirpation in embryos and larvae of different ages. J. Exp. Zool., 107: 217-267.

1954 Regeneration of the lens in the amphibian eye. Quart. Rev. Biol., 29: 1-46.

1971 DNA synthesis and the incorporation of la. beled iris cells into the lens during lens regeneration in adult newts. Develop. Biol., 24: 535-558.

Sato, T, 1940 Vergleichende Studien uber die Geschwindigkeit der Wolffschen Linsenregeneration bei Triton taeniatus und bei Diemictylus pyrrhogaster. Arch. Entwmech. Org., 140: 573-613.

Stone, L. S., and H. Steinitz 1953 The regeneration of lenses in eyes with intact and regenerating retina in adult Triturus v. viridescens. J. Exp. Zool., 124: 435-467.

Yamada, T. 1967 Cellular and subcellular events in Wolffian lens regeneration. Curr. Top. Develop. Biol., 2: 247-283.

- 1972 Control mechanisms in cellular metaplasia. Proc. 1st Int'l. Conf. Cell Differentiation. Harris et al., eds. Munksgaard, Copenhagen, pp. 56-60.

- 1977 Control mechanisms in cell-type conver. sion in newt lens regeneration. Mon. Develop. Biol., Vol. 13. A. Wolsky, ed. Karger, Basel.

Yamada, T., and M. Roesel 1964 Effects of Acintinomycin $\mathrm{D}$ on the lens regenerating system. J. Embryol. Exptl. Morphol., 12: 713-725.

Zalokar, M. 1944 Contribution a l'étude de la régénération du cristallin chez le Triton. Rev. Suisse Zool., 51: 443-521. 